\title{
Scaling of the Resolving Power and Sensitivity for Planar FAIMS and Mobility-Based Discrimination in Flow- and Field-Driven Analyzers
}

\author{
Alexandre A. Shvartsburg and Richard D. Smith \\ Biological Sciences Division, Pacific Northwest National Laboratory, Richland, Washington, USA
}

Continuing development of the technology and applications of field asymmetric waveform ion mobility spectrometry (FAIMS) calls for better understanding of its limitations and factors that govern them. While key performance metrics such as resolution and ion transmission have been calculated for specific cases employing numerical simulations, the underlying physical trends remained obscure. Here we determine that the resolving power of planar FAIMS scales as the square root of separation time and sensitivity drops exponentially at the rate controlled by absolute ion mobility and several instrument parameters. A strong dependence of ion transmission on mobility severely discriminates against species with higher mobility, presenting particular problems for analyses of complex mixtures. While the time evolution of resolution and sensitivity is virtually identical in existing FAIMS systems using gas flow and proposed devices driven by electric field, the distributions of separation times are not. The inverse correlation between mobility (and thus diffusion speed) and residence time for ions in field-driven FAIMS greatly reduces the mobility-based discrimination and provides much more uniform separations. Under typical operating conditions, the spread of elimination rates for commonly analyzed ions is reduced from $>5$ times in flow-driven to 1.6 times in field-driven FAIMS while the difference in resolving power decreases from $\sim 60 \%$ to $\sim 15 \%$. (J Am Soc Mass Spectrom 2007, 18, 1672-1681) (c) 2007 American Society for Mass Spectrometry

$\mathrm{F}$ ield asymmetric waveform ion mobility spectrometry (FAIMS) has emerged as a powerful new analytical technique [1-20]. All IMS methods separate ions using their transport in a gas under the influence of electric field [21]: conventional IMS is based on absolute ion mobility $(K)$ at particular field intensity $(E)$ and FAIMS works with the difference between $K$ at high and low E. So FAIMS is also known as differential mobility spectrometry (DMS) [11, 12]. The value of $K$ for all ions increases or decreases at sufficiently high $E$, depending on the interaction potentials with gas molecules. As those potentials differ for different ions, the $K(E)$ function is characteristic of the species and, in principle, any two could be distinguished by FAIMS.

Separation parameters in FAIMS are largely independent of the ion mass / charge state $(\mathrm{m} / \mathrm{z})$ ratio, which renders FAIMS substantially orthogonal to mass spectrometry (MS) and makes FAIMS/MS a powerful method of broad utility. The separation power of FAIMS/MS could be augmented by coupling to conventional IMS providing 2-D separations [17, 18] before MS and/or pre-ionization stages such as liquid or gas chromatog-

Address reprint requests to Dr. R. D. Smith, Biological Systems Analysis and Mass Spectrometry, Biological Sciences Division, Pacific Northwest National Laboratory, 3335 Q Ave. (K8-98) P.O. Box 999, Richland, WA 99352, USA. E-mail: rds@pnl.gov raphy (LC $[14,16,20]$ or GC $[5,8,9])$ or capillary electrophoresis (CE) [10]. The operational simplicity, small size, and low cost make FAIMS attractive for field analyses that require rugged, portable, inexpensive sensors. Recent commercialization of FAIMS technology, alone and in conjunction with LC/MS or GC, has enabled its expansion into proteomics [15-17], structural biology $[13,18,19]$, pharmaceutical analyses $[10,20]$, environmental monitoring $[4,14]$, and security applications such as detection and classification of fire sources [8], explosives [9, 11], chemical warfare agents [9], illicit drugs [9], and bacterial pathogens [12].

Ions in FAIMS are separated using a periodic asymmetric field $E_{\mathrm{D}}(t)$ that comprises short segments of high $E$ and long segments of low $E$ of opposite polarity such that the average $E$ is zero but absolute positive and negative $E$ are unequal [1]. Hypothetical ions with constant $K$ would oscillate in that field with no separation. The actual displacements $\Delta x$ during $\{E>0\}$ and $\{E<0\}$ do not cancel exactly and ions drift in the direction of high $E$ when $K$ (high $E)>K($ low $E$ ) and low $E$ otherwise (Figure 1a). In practice, $E_{D}(t)$ is established in an analytical gap between two electrodes carrying a waveform $V_{\mathrm{D}}(t)$. Ions injected into the gap would travel toward either electrode and be neutralized. The velocity of that motion would depend on $K(E)$ of the ion and 
(a)

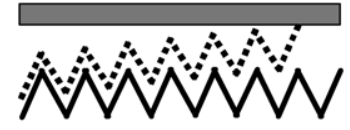

(b)

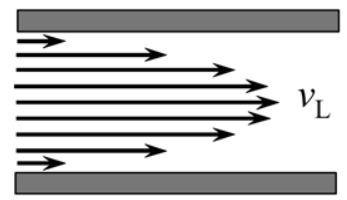

(c)

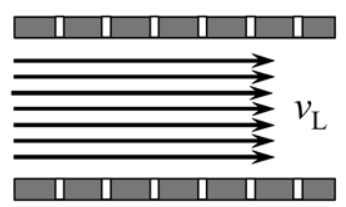

(d)

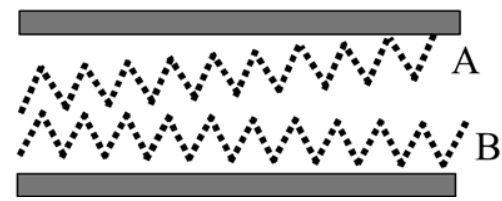

Figure 1. Fundamentals of FAIMS: (a) trajectory of an ion in the gap with $E_{\mathrm{C}}$ (solid line) and without $E_{\mathrm{C}}$ (dashed line), (b) flow-FAIMS with parabolic profile of ion velocity $v_{\mathrm{L}}$ across the gap, (c) field-FAIMS with uniform $v_{\mathrm{L}}$ (d) increase of resolving power at longer separation time (Species A that would pass a shorter analyzer in (a) is filtered out, Species B with a smaller difference between correct and actual $E_{\mathrm{C}}$ still passes but would be removed in a yet longer FAIMS)

thus differ for different species. For a particular species, this drift could be offset by a motion caused by constant "compensation field" $E_{\mathrm{C}}$ due to "compensation voltage" (CV) loaded on one of the electrodes [1]. This brings a subset of ions into equilibrium and allows it to pass the gap while others are filtered out; scanning CV reveals the spectrum of ions present.

Numerous $E_{\mathrm{D}}(t)$ profiles are possible and several have been considered in theory and experiment [22]. The ideal rectangular [22] $E_{\mathrm{D}}(t)$ with 2:1 ratio of high and low $E$ is commonly approximated by the waveform comprising two harmonics with 2:1 frequency ratio:

$$
E_{\mathrm{D}}(t)=E_{\max }\left[f \sin \left(2 \pi w_{c} t\right)+\sin \left(4 \pi w_{c} t-\pi / 2\right)\right] /(f+1)
$$

where $E_{\max }$ is the peak amplitude ("dispersion field"), $w_{c}$ is the frequency, $t$ is time, and $f$ controls the $E_{\mathrm{D}}(t)$ profile. The value of $f=2$ is close to optimum [22] and has been adopted in systems such as TSQ Quantum FAIMS/MS (ThermoFisher) [20] and SDP-1 and microAnalyzer DMS (Sionex) [23, 24].

In present FAIMS devices [1], ions are pushed through the gap by gas flow (Figure 1b). An alternative to that "flow-FAIMS" would be "field-FAIMS" with electric field $\left(E_{\mathrm{L}}\right)$ along the gap $[25,26]$, for example created by applying a ladder of dc voltages superposed on $V_{\mathrm{D}}(t)$ and /or CV to segmented electrodes (Figure 1c). Such addition of $\mathrm{rf}$ waveform to de voltage gradient is common in MS and IMS instruments including segmented quadrupoles [27, 28] and ion funnels [29-31]. However, the concept was not implemented for FAIMS, perhaps because no advantage over the existing flow approach was apparent.

While FAIMS analyses are based on $E_{C}$, the ion oscillation amplitude $\Delta x$ is proportional to $K$ [24]. Hence ions with higher $K$ come closer to electrodes and are more likely to be destroyed, which reduces the transmission efficiency $(s)$ through the gap and narrows the $E_{C}$ range for ion passage. Thus, other factors being equal, $s$ decreases and resolution improves for ions with higher $K$. For example, in planar FAIMS typically operated at $\sim 70-80$ Townsend (Td) or $E \sim 17-20$ $\mathrm{kV} / \mathrm{cm}$ at temperature $(T)$ of $298 \mathrm{~K}$ and pressure $(P)$ of $1 \mathrm{~atm}, \mathrm{~s}$ for xylene ${ }^{+}\left(\mathrm{H}_{2} \mathrm{O}\right)_{\mathrm{n}}$ ions exceeds that for $\mathrm{H}_{3} \mathrm{O}^{+}\left(\mathrm{H}_{2} \mathrm{O}\right)_{n}$ by $\sim 10$ times despite a small difference between their $K$ values [24].

A balance between sensitivity and resolving power $(R)$ is inherent in ion filtering methods, including quadrupole MS to which FAIMS is often compared [22, 32]. For FAIMS, the balance may be controlled by adjusting $w_{c}$ or gap width $(g)$ [22], changing the gas [22], or scaling the frequency or amplitude of a low-frequency "ripple" [33] added to $E_{\mathrm{D}}(t)$. In any case, the balance depends on $K$ and thus is nonuniform over an ion mixture. That is critical because mixtures analyzed by FAIMS often span a broad range of $K$. In biological analyses, the difference is by factors of $\sim 2.5$ for tryptic peptide ions generated by ESI [34] or MALDI [35], $>3$ for electrosprayed combinatorial peptide libraries [36], and $>5$ for a more diverse set comprising peptide, nucleotide, and lipid ions extracted from tissues using MALDI $[37,38]$. In security applications, mobilities of common chemical warfare agents and their environmental degradation products differ by $>2$ times $[39,40]$. So, to detect or quantify all species, $R$ in flow-FAIMS must be set to get sufficient signal for the ion with highest $K$ of those possibly present. This decreases the resolution and specificity for other ions. To limit the loss of overall peak capacity, one may need to acquire full CV scans for several $R$ settings (since $E_{C}$ and $K$ are not related directly). The discrimination in ion transmission through FAIMS based on $K$ also complicates quantification and, because of FAIMS charge capacity limitations [32], restricts the dynamic range of detection.

With curved electrodes such as coaxial cylinders or concentric spheres, the field in the gap is inhomogeneous [41]. Depending on the $E_{\mathrm{D}}(t)$ polarity and form of $K(E)$, that causes ions to focus to or defocus away from the gap median $[19,22,24,32,41]$, with $s$ respectively increasing or decreasing (often to zero). Of course, one chooses the appropriate polarity for ions of interest. However, focusing also expands the range of $E_{\mathrm{C}}$ allow- 
ing ions to be stable in the gap, which broadens FAIMS peaks. The focusing power depends on the form of $K(E)$ and thus preferentially increases the signal and decreases resolution for some ions [22]. In general (but not always), the effect is stronger for ions with higher $\left|E_{C}\right|$. For example, for peptide ions in $\mathrm{N}_{2}$ or $1: 1 \mathrm{He} / \mathrm{N}_{2}$, the $\left|E_{C}\right|$ rises in the $z=1,2,3$ sequence and the transmission efficiency and peak width in cylindrical FAIMS increase in parallel $[3,15,16]$. In planar FAIMS, homogeneous electric field allows no ion focusing and the $K(E)$ profile affects neither resolution nor sensitivity $[19,24]$, but the discrimination based on absolute $K$ remains.

The ion transmission and peak shapes in flow- and field-FAIMS were modeled employing molecular dynamics, with results validated by experiment [19, 22, 32, 33]. For example, the predicted increase of FAIMS resolving power with gap curvature decreasing toward a planar geometry had guided the development of high-resolution FAIMS [19]. However, previous studies focused on single ion species and did not address mixtures and issues of ion discrimination. That is done here using mathematical derivations and simulations that establish the scaling laws for resolution and sensitivity of flow- and field-planar FAIMS as a function of ion mobility and separation time. In particular, we show that fieldFAIMS will greatly reduce the mobility-based discrimination, providing far more uniform separations of complex ion mixtures.

\section{Crucial Distinction Between Separations in Flow- and Field-FAIMS}

The basic difference between flow- and field-FAIMS stems from different distributions of ion residence time $\left(t_{\text {res }}\right)$. In the former, the longitudinal velocity of ions $\left(v_{\mathrm{L}}\right)$ equals local gas flow velocity that has a quasi-parabolic profile across the gap because steady-state boundary conditions for a gas of finite viscosity require $v_{\mathrm{L}}=0$ on electrode surfaces (Figure 1b) [33]. In the latter, a uniform $E_{\mathrm{L}}$ would result in equal $v_{\mathrm{L}}$ and $t_{\text {res }}$ for all trajectories of a particular species (ignoring longitudinal diffusion) (Figure 1c). The profile of $t_{\text {res }}$ across the gap is material because the ion path through FAIMS depends on $E_{C}$ : an ion passes near the gap median at $E_{C}$ of a peak apex in $C V$ spectrum and closer to electrodes at $E_{C}$ corresponding to peak shoulders [33]. Hence, in flowFAIMS, ions with $E_{C}$ away from peak centers have longer $t_{\text {res }}$, which facilitates their elimination by both diffusion and directed filtering. This effect makes features narrower in flow- than in field-FAIMS, but the difference is only a few percent [33]. Diffusion of ions along $v_{\mathrm{L}}$ produces a distribution of $t_{\text {res }}$ around mean values, but that applies to both cases and does not really affect that small difference [33].

For mixtures, a far more important distinction is that in flow-FAIMS all components have equal mean $v_{\mathrm{L}}$ and $t_{\text {res, }}$ while in field-FAIMS

$$
t_{\text {res }}=L / v_{\mathrm{L}}=L /\left(K E_{\mathrm{L}}\right)
$$

where $L$ is the gap length. As longer separation leads to higher FAIMS resolution [19], the increase of $t_{\text {res }}$ at lower $K$ by eq 2 may offset the decrease of resolution at fixed $t_{\text {res }}$ (Figure 1d). To evaluate the ramifications of that previously unrecognized aspect for reducing ion discrimination, we need to establish how the FAIMS resolution and sensitivity evolve as a function of $t_{\text {res. }}$.

\section{Scaling of Resolution and Sensitivity in Flow-FAIMS}

\section{Fundamentals}

In planar FAIMS, ions freely diffuse in all directions until hitting an electrode. The solution of diffusion equation with zero boundary conditions is [24]:

$$
s=\exp \left(-D \pi^{2} t / g^{2}\right)
$$

Here $\mathrm{D}$ is the diffusion coefficient related to mobility by the Einstein equation

$$
D=k_{\mathrm{B}} K T / q
$$

where $k_{\mathrm{B}}$ is the Boltzmann constant and $q$ is the absolute ion charge. As expected, ions with higher $K$ are eliminated from FAIMS faster. Equation 3 is rigorous only in the limit of $\Delta x / g=0$ approached at high $w_{c}$, large $g$, or small $K$. In reality, oscillations effectively narrow the gap and $s(t)$ drops faster than given by eq 3 . That was accounted for [24] by interpreting $g$ in eq 3 as the effective gap width, $g_{\mathrm{e}}=g-\Delta x$. The value of $\Delta x$ is:

$$
\Delta x=\frac{K(0)}{2} \int_{0}^{t_{p e r}}\left|E_{\mathrm{D}}(t)\right| d t
$$

where $t_{\text {per }}$ is the $E_{\mathrm{D}}(t)$ period. Equation 5 is integrable for $E_{\mathrm{D}}(t)$ of eq 1 with any $f$, for $f=2$ :

$$
\begin{aligned}
\Delta x & =\left[\left(3^{1 / 4}+3^{-1 / 4}\right) /\left(2^{3 / 2} \pi\right)\right] K(0) E_{\max } t_{\mathrm{per}} \\
& \approx 0.2336 K(0) E_{\max } / w_{\mathrm{c}}
\end{aligned}
$$

Hence ion transmission through FAIMS decreases for ions with higher $K$ because of both increasing $D$ and decreasing $g_{\mathrm{e}}$.

At high $E$ where the ion drift velocity is comparable to thermal velocities of gas molecules, the diffusion becomes anisotropic: ions spread faster along $\mathbf{E}$ than in the orthogonal plane [42]. The diffusion along E (i.e., perpendicular to FAIMS electrodes) is governed by the longitudinal diffusion coefficient, $D_{\mathrm{L}}$. Equation 4 is modified as [32]: 


$$
D_{L}(E / N)=\frac{k_{B} T_{L} K(E / N)}{q}\left(1+\frac{\partial \ln K(E / N)}{d \ln (E / N)}\right)
$$

Here $N$ is the number density of gas molecules and $T_{\mathrm{L}}$ is the "longitudinal temperature" [32]:

$$
T_{L}(t) \cong T+\left(1+\frac{2}{2+M / m}\right) \frac{M K^{2}(0) E^{2}(t)}{3 k_{B}}
$$

where $M$ is the mass of gas molecule. The contribution of the second term in eq 7 is normally small both in absolute sense and compared to that of the second term of eq 8, and may for now be omitted. The relevant quantity here is the mean $D_{\mathrm{L}}$ over $E(t)$ cycle, so $E^{2}(t)$ in eq 8 should be replaced by $\left\langle E^{2}(t)\right\rangle$. For the $E(t)$ of eq 1 with $f=2$, one finds $\left\langle E^{2}(t)\right\rangle=5 E_{\max }^{2} / 18$ and:

$$
\left\langle D_{L}\right\rangle \cong D+\left(1+\frac{2}{2+M / m}\right) \frac{5 M K^{3}(0) E_{\max }^{2}}{54 q}
$$

Hence $\left\langle D_{\mathrm{L}}\right\rangle$ grows as a function of $K$ faster than linearly, which augments the disproportional elimination of ions with higher $K$. For small ions with high $K$, the effect may be dramatic. For instance [43], $K(0)=2.9 \mathrm{~cm}^{2} /$ $(\mathrm{V} \times \mathrm{s})$ for ${ }^{35} \mathrm{Cl}^{-}$in ambient air. In FAIMS operated at

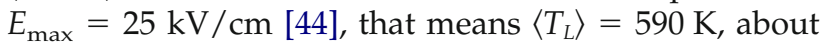
doubling the diffusion speed over that at low $E$.

While ion transmission through planar FAIMS drops for longer separations, the resolution improves [19]. Here we define the resolving power as $R=E_{\mathrm{C}} / w$, where $w$ is the full peak width at half maximum. Ignoring the diffusion, that was quantified as [24]:

$$
R=K E_{\mathrm{C}} t_{\text {res }} / g
$$

where $g$ should also be $g_{\mathrm{e}}=g-\Delta x$. Thus resolution should increase at higher $K$, because of both faster elimination of species with improper $E_{C}$ and greater amplitude of oscillations that effectively narrows the gap.

\section{Simulation Procedure}

Modeling has employed the established treatment [32]. Earlier simulations involved $\sim 10^{2}-10^{3}$ ions [32], producing good statistics for high $s$ but not in a low sensitivity/high-resolution regime when few ions survived. Here we start with $\sim 10^{4}-10^{5}$ ions, yielding sufficient statistics for $s$ as low as $\sim 0.2 \%$ (that is perhaps less than acceptable in practice). Unlike in previous studies where FAIMS separations were modeled for specific $t_{\text {res }}$ set by the gap length and flow speed [22, 32, 33] (usually $0.2 \mathrm{~s}$ ), here simulations go on till all ions are lost, which may take $>1 \mathrm{~s}$. We also extend calculations to $w_{\mathrm{c}}=2.5 \mathrm{MHz}$, requiring simulations for $>3 \times 10^{6}$ cycles versus $<1.5 \times 10^{5}$ in previous work. The drastic increase of computational cost has been offset somewhat by ignoring Coulomb repulsion. Hence present modeling assumes a modest ion current density, which is reasonable for planar FAIMS where the lack of ion focusing precludes high charge density. In the absence of space charge, all ions move independently and absolute or relative density of various ion species does not affect the simulation results. The non-uniformity of flow velocity in flow-FAIMS, shown to have little impact [33], was also neglected.

Singly-charged ions are represented by species with $m=35,130,260$, and $609 \mathrm{Da}$ and $K$ (in $\mathrm{N}_{2}$ or air at 298 $\mathrm{K})$ of $3.0,2.18,1.50$, and $0.9 \mathrm{~cm}^{2} /(\mathrm{V} \times \mathrm{s})$, respectively. These properties are close to those of, respectively, $\mathrm{Cl}^{-}$, leucine $(1+)$, 3,4-benzpyrene $(1+)$, and reserpine $(1+)$ $[22,43,45]$. The ion mass has only a minor effect through the parenthetical term in eq 9 that equals 1.7-2.0 for all $m>35 \mathrm{Da}$. To elicit the dependence of FAIMS response on absolute $K$ only, we assumed equal $K(E)$ profiles for all ions, defined by:

$$
K(E)=K(0) \times\left(1+a E^{2}+b E^{4}\right)
$$

where $a=5.43 \times 10^{-6} \mathrm{Td}^{-2}$ and $b=-1.85 \times 10^{-10}$ $\mathrm{Td}^{-4}$. These values are for (Leucine $\left.-\mathrm{H}\right)^{-}$, the test case in preceding FAIMS simulations $[19,22,32,33]$. The instrumental parameters were $g=0.2 \mathrm{~cm}$ and $E_{\max }=$ $16.5 \mathrm{kV} / \mathrm{cm}$, which were typical for much experimental and modeling work. Under those conditions, $E_{\mathrm{C}}=-36$ $\mathrm{V} / \mathrm{cm}$ for all ions [22].

\section{Results}

The findings for $w_{\mathrm{c}}=210 \mathrm{kHz}$ (used in some FAIMS systems and most previous modeling) [22, 32, 33] are presented in Figure 2. In agreement with eq 3, $s(t)$ is an exponent for all $K$ (Figure 2a). The coefficients $A_{1}$ in $\left\{\operatorname{Ln}(s)=-A_{1} t\right\}$ are compared with those from eqs 3,5 , 9 in Table 1. At the lowest $K=0.9 \mathrm{~cm}^{2} /(\mathrm{V} \times \mathrm{s})$, the mean $D_{\mathrm{L}}$ exceeds $D$ by $<5 \%$ and $A_{1}$ given by either eq 4 or eq 9 is close to simulation. For higher $K$, the simulated $A_{1}$ increasingly exceed those by eq 4 but match those by eq 9 within the uncertainty of modeling $(<3 \%)$. Thus eqs 3, 5, 9 allow accurate prediction of ion transmission through planar FAIMS under various conditions.

As expected, the resolution improves for longer residence times and higher $K$ (Figure $2 b$ ). However, the $R(t)$ dependences are not linear: the $R / t$ values for all $K$ are close to those by eq 10 at lowest $t_{\text {res }}$ but decrease at higher $t_{\text {res }}$, falling $\sim 50 \%$ to $60 \%$ short at highest $t_{\text {res }}$ considered (Figure 2c). As eq 3 that incorporates diffusion is apparently correct, the failure of eq 10 must be due to its neglect. Free diffusion of an initially point-like ion packet (in one dimension defined by coordinate $x$ ) is governed by [42]:

$$
N_{1}(x, t)=\frac{S}{2 \sqrt{\pi D t}} \exp \frac{-x^{2}}{4 D t}
$$

where $S$ is the total number of ions and $N_{\mathrm{I}}$ is their density, and 

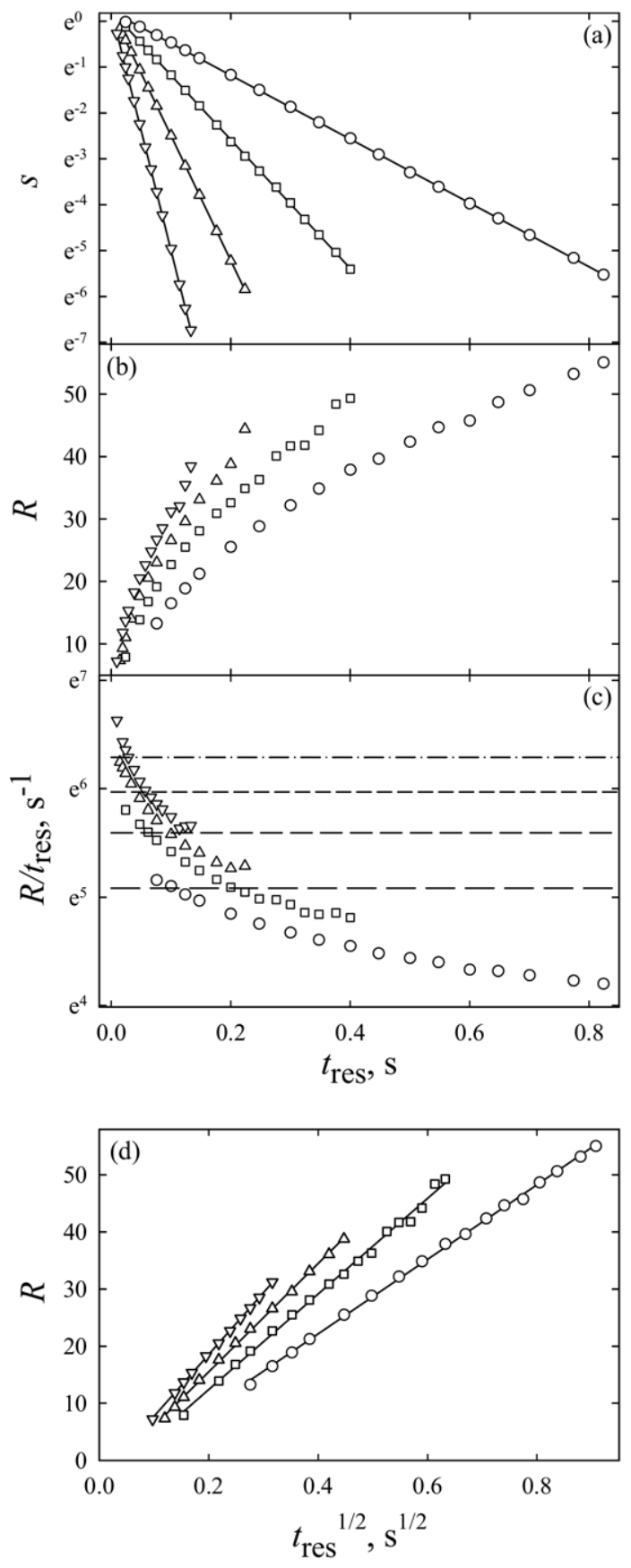

Figure 2. Time-evolution of transmission efficiency (a) and resolving power $(\mathbf{b})-(\mathbf{d})$ in planar flow-FAIMS for ions with different mobilities, at $w_{\mathrm{c}}=210 \mathrm{kHz}$. The vertical axes are on the log scale in (a), (c) and linear scale in (b), (d). Symbols are from simulations, solid lines (a), (d) correspond to linear regressions for each $K$, and dashed lines (c) are values by eq 10. Data are shown for $K\left[\right.$ in $\left.\mathrm{cm}^{2} /(\mathrm{V} \times \mathrm{s})\right]$ of $0.9(\bigcirc$, long dash), 1.5 ( $\square$, medium dash), 2.18 ( $\triangle$, short dash), and $3.0(\nabla$, dash-dot). The sensitivity and resolving power scale with time as the inverse exponent (a) and square root (d), respectively.

$$
w=2 \sqrt{D t \ln 2}
$$

As the separation distance between two analytes is proportional to $t_{\text {res }}$, the (diffusion-limited) resolving power of 1-D separations should scale as $t_{\text {res }}^{1 / 2}$. That is commonly found for both liquid and gas-phase methods, including conventional IMS or CE where $[42,46]$ :

$$
R=\sqrt{q U /\left(k_{b} T 1 \mathrm{n} 2\right)} / 4
$$

where $U$ is the drift voltage, or, using eq 4 :

$$
R=E q \sqrt{D t / 1 \mathrm{n} 2} /\left(4 k_{B} T\right)
$$

Simulations (Figure 2d) show that in planar FAIMS, $R$ for all $K$ also scale with $t_{\text {res }}{ }^{1 / 2}$ (after the initial induction period). This evidences the key role of diffusion: in essence, FAIMS filters out ions with wrong $E_{C}$ not by forcing them into an electrode directly, but by accelerating their diffusional loss via bringing them closer to an electrode. One might expect $R$ to scale as $\left\langle D_{\mathrm{L}} t_{\text {res }}\right\rangle^{1 / 2}$ given the dimensionality of variables and considering eqs 9 and 15 , but the dependence on $\left\langle D_{\mathrm{L}}\right\rangle$ in simulations is clearly weaker than $\left\langle D_{\mathrm{L}}\right\rangle^{1 / 2}$ and close to $\left\langle D_{\mathrm{L}}\right\rangle^{0.3}$ (Table 1).

To explore the scaling of resolution and sensitivity in planar FAIMS further, we have performed simulations for $K$ of 0.9 and $3.0 \mathrm{~cm}^{2} /(\mathrm{V} \times \mathrm{s})$ over $w_{\mathrm{c}}=100 \mathrm{kHz}-2.5$ $\mathrm{MHz}$. This broad range encompasses the values adopted in all FAIMS systems built to date, e.g., 128$147 \mathrm{kHz}$ in the first commercial unit by Mine Safety Appliances Co. (MSA) [47], $167 \mathrm{kHz}$ in the GC-FAIMS by DTIIGE [9], $750 \mathrm{kHz}$ in Selectra by Ionalytics [1], TSQ Quantum [20], and the PNNL high-resolution planar analyzer [19], and 1.2-2.0 MHz in miniaturized planar FAIMS [24, 48, 49] (the MSA, DTIIGE, Selectra, and TSQ devices have cylindrical geometries.) In all cases, the modeled $s(t)$ remains an exponential (Figure 3a) in excellent agreement with eqs 3,9 (Table 1). As expected, the ion transmission drops at higher $K$, particularly at low $w_{c}$ values where $\Delta x$ is a significant fraction of $g$. The resolving power is proportional to $t^{1 / 2}$ at all $K$ but hardly depends on $w_{\mathrm{c}}$ (Figure $3 \mathrm{~b}$, Table 1 ). This is a bit counterintuitive as raising the amplitude of ion oscillations in the $E_{\mathrm{D}}(t)$ cycle should effectively constrain the gap and increase $R$ [22]. However, unlike for the cylindrical geometry, the resolution of planar FAIMS with a reasonably tight gap depends on its width only slightly [19] and the data in Figure 3b are consistent with previous findings [19]. While a priori derivation of $R(t)$ in the manner of $s(t)$ is left to future work, the present determination of its scaling with $t^{1 / 2}$ and (virtual) independence of $w_{\mathrm{c}}$ will prove useful in the following.

The discrimination against ions with higher $K$ is apparent at any $w_{\mathrm{c}}$ (Figures $2 \mathrm{a}$ and $3 \mathrm{a}$ ) but diminishes at higher $w_{\mathrm{c}}$ because of $\Delta x / g$ decreasing by eq 6: the spreads of $A_{1}$ values over the $K=0.9-3.0 \mathrm{~cm}^{2} /(\mathrm{V} \times \mathrm{s})$ range are $18,7.5,5.4$, and 4.9 times at $w_{\mathrm{c}}=100,210,750$, and $2500 \mathrm{kHz}$, respectively (Table 1). In the limit of infinite $w_{c}$, the spread of $A_{1}$ obtained using eqs $3,5,9$ is 4.8 times. Hence, while raising the waveform frequency or widening the gap reduces mobility-based discrimination in FAIMS, it remains severe even in the hypothetical limit of infinite frequency and/or gap width. As 
Table 1. Coefficients for $s(t)=\left\{\exp \left(-A_{1} t\right)+\mathrm{C}\right\}$ and $R(t)=\left\{A_{2} t^{1 / 2}+\mathrm{C}\right\}$

\begin{tabular}{|c|c|c|c|c|c|c|c|c|c|c|c|c|}
\hline \multirow{2}{*}{$\frac{w_{\mathrm{c}^{\prime}} \mathrm{kHz}}{\overline{K, \mathrm{~cm}^{2} /(\mathrm{V} \times \mathrm{s})}}$} & \multicolumn{4}{|c|}{210} & \multicolumn{2}{|c|}{100} & \multicolumn{2}{|c|}{150} & \multicolumn{2}{|c|}{750} & \multicolumn{2}{|c|}{2500} \\
\hline & 0.9 & 1.5 & 2.18 & 3.0 & 0.9 & 3.0 & 0.9 & 3.0 & 0.9 & 3.0 & 0.9 & 3.0 \\
\hline \multicolumn{13}{|l|}{$A_{1}, \mathrm{~s}^{-1}$} \\
\hline Simulated $^{a}$ & 6.9 & 14 & 27 & 52 & 8.6 & 158 & 7.6 & 72 & 6.1 & 33 & 5.8 & 28 \\
\hline Eqs $(3,4)$ & 6.8 & 13 & 22 & 36 & 8.4 & 107 & 7.3 & 50 & 6.0 & 22 & 5.8 & 20 \\
\hline Eqs $(3,9)$ & 7.1 & 14 & 27 & 52 & 8.7 & 154 & 7.6 & 72 & 6.2 & 32 & 6.0 & 29 \\
\hline \multicolumn{13}{|l|}{ Scaling of ${ }^{\mathrm{b}} A_{2}$} \\
\hline Simulated $^{\mathrm{a}}$ & 1.0 & 1.22 & 1.39 & 1.59 & 1.02 & 1.57 & 1.02 & 1.58 & 1.03 & 1.64 & 1.05 & 1.62 \\
\hline as $\left\langle D_{\mathrm{L}}\right\rangle^{1 / 2}$ & 1.0 & 1.37 & 1.74 & 2.19 & 1.0 & 2.19 & 1.0 & 2.19 & 1.0 & 2.19 & 1.0 & 2.19 \\
\hline as $\left\langle D_{\mathrm{L}}\right\rangle^{0.3}$ & 1.0 & 1.21 & 1.39 & 1.60 & 1.0 & 1.60 & 1.0 & 1.60 & 1.0 & 1.60 & 1.0 & 1.60 \\
\hline
\end{tabular}

a Values from linear regressions in Figure 2a and d, Figure 3.

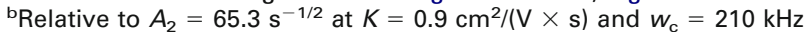

discussed above, the spread of factors $A_{2}$ in $R=A_{2} t^{1 / 2}$ over the range of $K$ studied is 1.6 times, regardless of $w_{\mathrm{c}}$.

\section{Reduction of Discrimination Using Field-FAIMS}

\section{Time Scaling of Resolution and Sensitivity}

The $s(t)$ and $R(t)$ functions in field-FAIMS are identical to those in flow analyzers, except the $t$ axis for each ion is rescaled according to eq 2 . The resulting curves for different $K$ (Figure 4) lie much closer than those in Figures 2 and 3 , evidencing a greatly reduced discrimination. For $s(t)$, the spreads of $A_{1}$ values over the range of $K=0.9-3.0 \mathrm{~cm}^{2} /(\mathrm{V} \times \mathrm{s})$ decrease by a factor of $3.0 / 0.9 \approx 3.3$ to $5.5,2.3,1.6$, and 1.5 times at $w_{\mathrm{c}}=100$, 210, 750, and $2500 \mathrm{kHz}$, respectively (Figure $4 \mathrm{a}$ and b). To clarify the origin of remaining discrimination, we combine eqs $2-4$ into:

$$
s=\exp \left(-\frac{\pi^{2} k_{B} T L}{q E_{L} g^{2}}\right)
$$

This expression contains neither $D$ nor $K$ and, had eq 3 been rigorous, no mobility-based discrimination would have existed. However, as discussed above, the variables $g$ and $T$ in eq 16 are actually $g_{\mathrm{e}}$ and $\left\langle T_{\mathrm{L}}\right\rangle$ that depend on $K$, hence ion discrimination in field-FAIMS. The first factor could be reduced in the same proportion as in flow-FAIMS by increasing $w_{\mathrm{c}}$ and/or $g$, as shown by simulations (Figure $4 \mathrm{~b}$ ). In the limit of $g_{\mathrm{e}}=g$, the spread of $A_{1}$ decreases to 1.4 times due to $\left\langle T_{\mathrm{L}}\right\rangle>T$. That factor cannot be reduced much and sets the minimum ion discrimination. Nonetheless, compression of the range of loss rates for common analytes from $\sim 5$ times in flow- to $\sim 1.5$ times in field-FAIMS (at practical $w_{\mathrm{c}} \sim 750$ $\mathrm{kHz}$ ) would be a major improvement.

The gain in uniformity of resolution provided by field-FAIMS (Figure $4 \mathrm{c}$ and $\mathrm{d}$ ) is even greater than that for sensitivity. The scaling of $t$ axis by eq 2 decreases the spread of $A_{2}$ at any $w_{\mathrm{c}}$ from 1.6 times to $<15 \%$, with the resolving power now increasing for ions with lower $K$ (Figure $4 \mathrm{~d}$ ). A variation of $R$ within $15 \%$ is close to typical random uncertainty in FAIMS experiments and would hardly be noted in practice. For example, $R(t)$ curves for $K=0.9,1.50,2.18$, and $3.0 \mathrm{~cm}^{2} /(\mathrm{V} \times \mathrm{s})$ could be fit using a single linear regression with $r^{2}=0.997$ (Figure 4c).
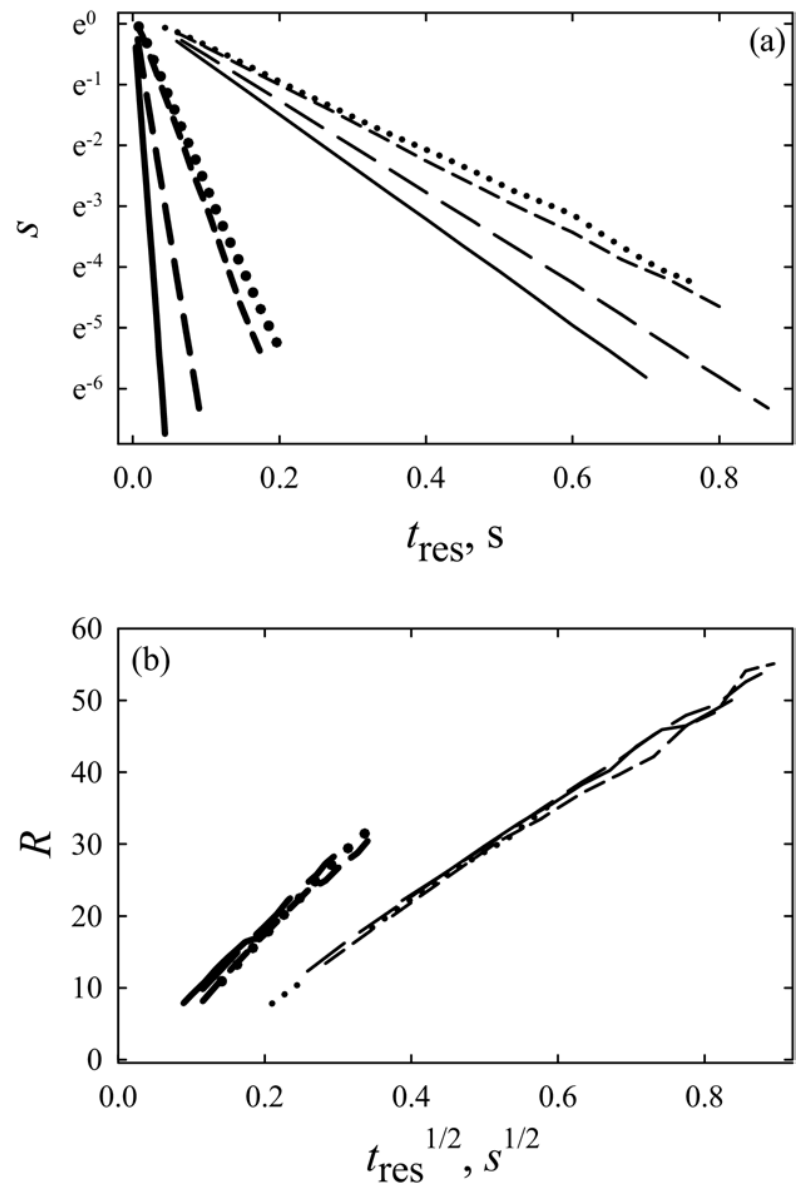

Figure 3. Time-evolution of transmission efficiency (a) and resolving power (b) over a range of waveform frequencies: $100 \mathrm{kHz}$ (solid lines), $150 \mathrm{kHz}$ (long dash), $750 \mathrm{kHz}$ (short dash), and 2.5 $\mathrm{MHz}$ (dotted). The vertical axes are on the log scale in (a) and linear scale in (b). Data are for $K=0.9 \mathrm{~cm}^{2} /(\mathrm{V} \times \mathrm{s})$ (thin lines) and $3.0 \mathrm{~cm}^{2} /(\mathrm{V} \times \mathrm{s})$ (thick lines). 

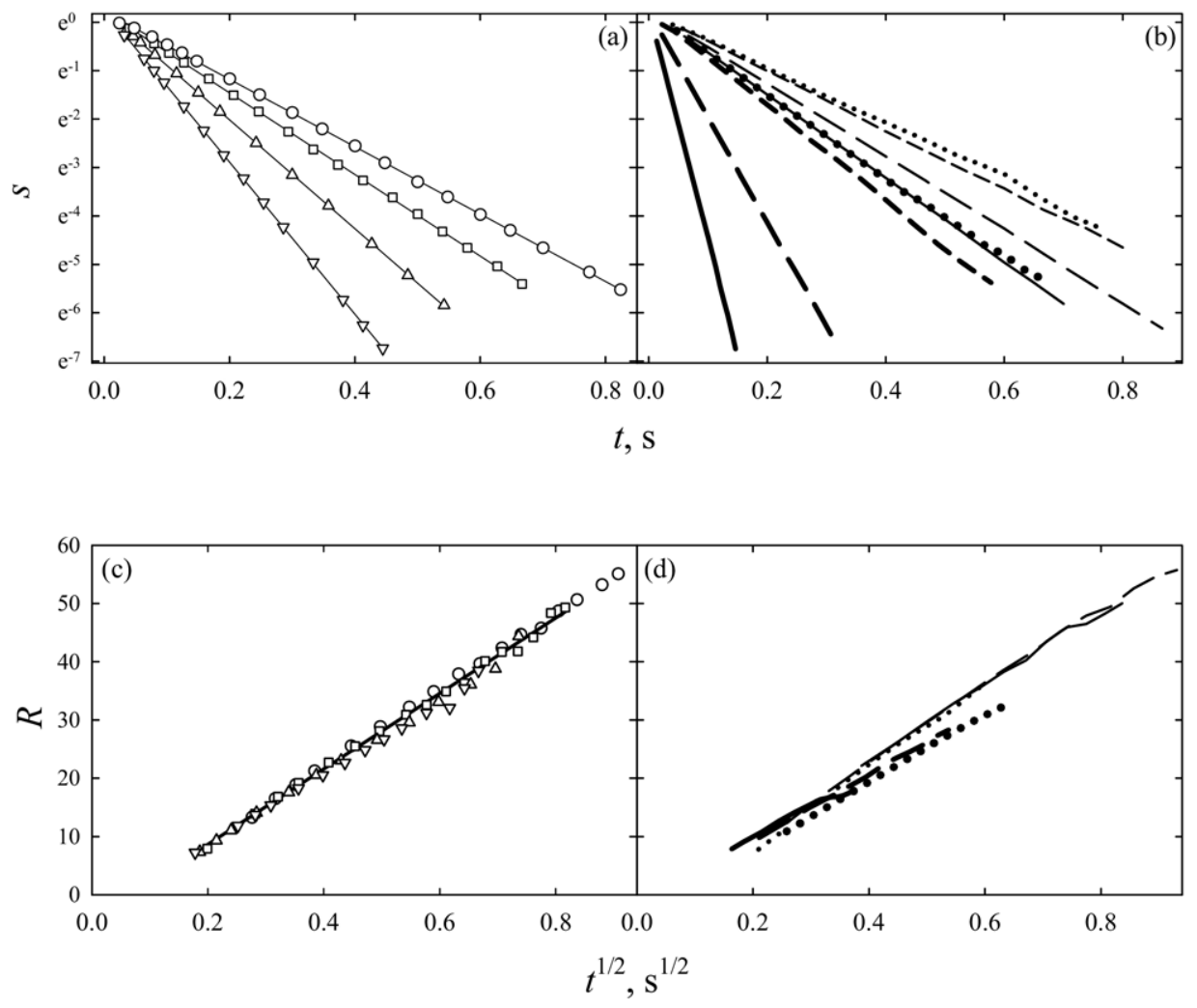

Figure 4. Time-evolution of transmission efficiency (a), (b) and resolving power (c), (d) in planar field-FAIMS, $t_{\text {res }}$ set for ions with the lowest $K=0.9 \mathrm{~cm}^{2} /(\mathrm{V} \times \mathrm{s})$. Panels $(\mathbf{a}),(\mathbf{c})$ and $(\mathbf{b}),(\mathbf{d})$ are parallel to Figures $2(\mathbf{a}),(\mathbf{d})$ and $3(\mathbf{a}),(\mathbf{b})$, respectively. The line in (c) is the first-order regression through all data.

\section{Resolution/Sensitivity Balance}

As with other analytical methods, one metric of FAIMS performance is the balance between resolution and sensitivity defined by $R / s$ diagrams $[19,33]$. A better $R / s$ balance, meaning a higher $R$ at equal $s$ for all $s$, is generally sought. The $R / s$ curves deduced from Figures 2 and 3 at any $w_{c}$ substantially depend on K (Figure 5). This may appear to contravene the notion that the value of $K$ affects the position on $R / s$ curves but not the curves themselves [33]. That is true when $t_{\text {res }}$ is fixed and $R$ is controlled by other means (e.g., changing the gap width or waveform frequency) [19, 32], while the $R / s$ balance in Figure 5 is adjusted by varying $t_{\text {res }}$. As ions with lower $K$ survive in FAIMS longer, the balance for them is better. However, real separations are subject to $t_{\text {res }}$ constraints.

The $R / \mathrm{s}$ curves for flow- and field-FAIMS are extracted from sets of $R / s$ graphs by connecting the points corresponding to equal $t_{\text {res }}$ and $t_{\text {res }}$ scaled by eq 2 , respectively (Figure 5). At any $w_{c}$, the curves have negative slopes (i.e., sensitivity drops with resolution improving at higher $K$ ) for flow-FAIMS but positive slopes (both $R$ and $s$ decrease at higher $K$ ) for fieldFAIMS (Figure 6). The spans of curves for same range of $K$ are shorter with field- than with flow-FAIMS, especially when the former is adjusted for reasonably high transmission efficiency $(>\sim 10 \%)$ expected in most analyses. This reflects a major reduction of discrimination in field-FAIMS. In its complete absence, the $R / s$ curves for field-FAIMS would have collapsed into points on the curves for flow analyzers at locations set by $K_{\text {ben }}$ - the value of $K$ given by eq 2 for $t_{\text {res }}$ of benchmark flowFAIMS and chosen $E_{\mathrm{L}}$. Because of residual discrimination, the curves for field-FAIMS retain a finite span, with $R / s$ balance worse than that for flow-FAIMS when $K>K_{\text {ben }}$ and better when $K<K_{\text {ben }}$ (Figure 6). That is easy to understand: ions with $K<K_{\text {ben }}$ spend longer time in field- than in flow-FAIMS, allowing a superior separation performance.

\section{Conclusions}

The scaling of resolving power and sensitivity of planar FAIMS has been determined using a priori derivations and numerical simulations. The ion transmission decays exponentially; the decay rate, described by the new formulas in full agreement with simulations, depends on ion mobility $(K)$, the FAIMS gap width, and the frequency, profile, and amplitude of asymmetric waveform. The resolving power $(R)$ increases as the square root of residence time $\left(t_{\text {res }}\right)$ under all conditions tried. These scaling laws allow predicting and optimizing many as- 


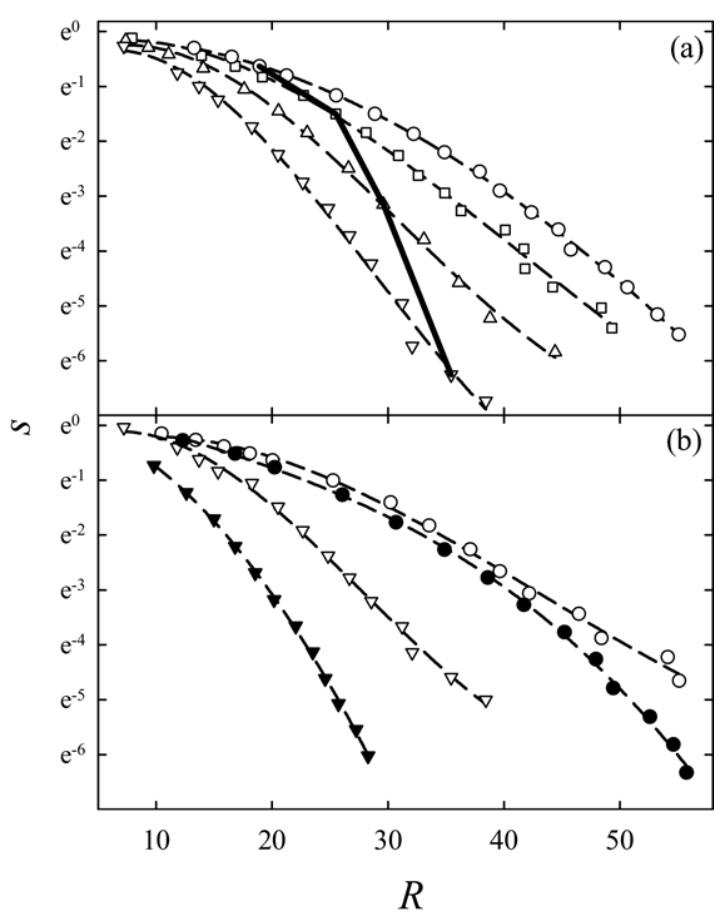

Figure 5. Resolution/sensitivity balance as a function of $t_{\text {res }}$ at $w_{\mathrm{c}}=210 \mathrm{kHz}(\mathbf{a}), 150 \mathrm{kHz}(\mathbf{b}),(\bullet, \nabla)$, and $750 \mathrm{kHz}(\mathbf{b}),(\bigcirc, \nabla)$; the $s$ axis is on the log scale. All symbols are as in Figure 2, dashed lines are third-order regressions through each curve. The solid line (a) is the $R / \mathrm{s}$ graph at fixed $t_{\text {res }}=124 \mathrm{~ms}$, obtained as described in the text.

pects of FAIMS performance without simulations and permit their validation when simulations are needed.

Though FAIMS filtering is based on the difference between $K$ in strong and weak electric field regardless of absolute $K$, the sensitivity of planar FAIMS depends on $K$. That causes harsh discrimination against ions with higher $K$, often up to their total elimination. The discrimination somewhat diminishes for greater waveform frequency $\left(w_{c}\right)$ and gap width $(g)$ but remains severe under any circumstances. This is a major problem with FAIMS analyses of complex mixtures (such as proteolytic digests) that typically comprise ions with a broad range of mobility.

This discrimination could be greatly reduced using FAIMS with ions propelled through the gap by electric field. In standard FAIMS employing gas flow to carry ions, all species have same $t_{\text {res }}$. In field-driven FAIMS, $t_{\text {res }}$ of an ion is proportional to $1 / \mathrm{K}$ : species with higher $K$ pass faster. This largely cancels the effect of $K$ on transmission at a fixed $t_{\text {res, }}$ producing much more uniform peak width and sensitivity for all ions. For example, the minimal spread of decay rates for ions with $K$ in the $0.9-3.0 \mathrm{~cm}^{2} /(\mathrm{V} \times \mathrm{s})$ range comprising most relevant species generated by ESI drops from $\sim 5$ in standard to $\sim 1.5$ in field-driven planar FAIMS. The spread of $R$ values is compressed by a similar factor, from $60 \%$ to $\sim 15 \%$; maximizing $w_{\mathrm{c}}$ and $g$ minimizes discrimination.
While the present modeling is for a bisinusoidal waveform used in most FAIMS systems, the mobilitybased discrimination and its suppression in field-driven FAIMS are fundamental to differential IMS and would occur with any waveform profile, including the clippedsinusoidal one adopted in some FAIMS analyzers [9, 47] and new asymmetric waveforms proposed for higherorder differential IMS (HODIMS) separations [50].

This physics is similarly manifested in drift-tube (DT) IMS, where $t_{\text {res }}$ for an ion is also proportional to $1 / K$ and packets of ions that diffuse faster have less time to expand. The difference is that the electric field in DT IMS is normally weak and the diffusion constant is rigorously proportional to $K$ by eq 4 , resulting in exactly equal $R$ for all $K$ [46]. In FAIMS, the speed of anisotropic high-field diffusion in a strong field of asymmetric waveform scales with $K$ more than linearly, while $t_{\text {res }}$ is determined by weak longitudinal field and still scales as $1 / K$. The mismatch causes residual discrimination in field-FAIMS. This also happens in high-field DT IMS where $R$ depends on $K$ and eqs 14,15 are modified [51].

Compared to a flow-driven FAIMS, a field-driven device is obviously a greater technical challenge from both mechanical and electrical viewpoints. So it is hardly surprising that, absent expected performance benefits, one has not yet been constructed. A major suppression of mobility-based discrimination revealed here is a substantial potential advantage of field-driven FAIMS. It will be particularly important for global separations of diverse samples, such as in proteomic and metabolomic discovery, but would also be of value for targeted analyses when the desired species has higher mobility than the interference or chemical noise in same CV range. Conversely, analyses where the target has a lower mobility than the "co-eluting" inter-

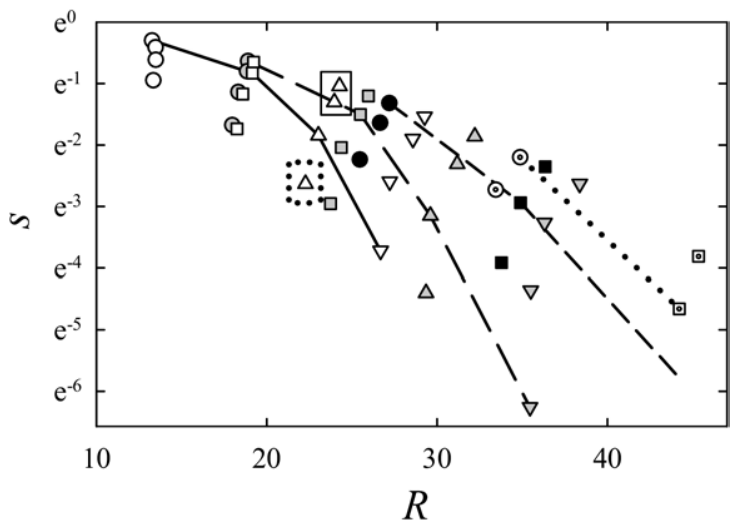

Figure 6. Resolution/sensitivity diagrams for flow-FAIMS (lines) and field-FAIMS (symbols) at $w_{\mathrm{c}}=210 \mathrm{kHz}$; the $s$ axis is on the log scale. Data are for $t_{\text {res }}=76 \mathrm{~ms}$ (solid lines, $O, \square, \triangle, \nabla$ ), $124 \mathrm{~ms}$ (long dash, $C, \triangle, \nabla, \square), 224 \mathrm{~ms}$ (short dash, $\bullet, \mathbf{\square}$ ), and $348 \mathrm{~ms}$ (dotted line and $\odot, \bullet)$. In field-FAIMS, $K_{\text {ben }}$ values are $0.9(\bigcirc, \odot, \bullet, \odot), 1.5(\square, \square$, $\mathbf{\square}, \bullet), 2.18(\triangle, \triangle)$, and $3.0 \mathrm{~cm}^{2} /(\mathrm{V} \times \mathrm{s})(\nabla, \nabla)$. The comparison of $R / \mathrm{s}$ balances for field- and flow-FAIMS is illustrated for $\left\{K_{\text {ben }}=2.18\right.$ $\left.\mathrm{cm}^{2} /(\mathrm{V} \times \mathrm{s}), t_{\text {res }}=76 \mathrm{~ms}\right\}$ : the balance in field-FAIMS is better for ions with $K=0.9$ and $1.5 \mathrm{~cm}^{2} /(\mathrm{V} \times \mathrm{s})$ (in the solid box) and worse for $K=3.0 \mathrm{~cm}^{2} /(\mathrm{V} \times \mathrm{s})$ (in the dashed box). 
ference may benefit from discrimination in flowFAIMS. Thus either flow- or field-FAIMS may be best for targeted applications, depending on the relative mobilities of target and interfering ions.

The mechanical package of field-FAIMS may still be operated as a flow-FAIMS, allowing rapid flexible switching between the two modes. The field- and flowdrives could also be combined in any proportion for optimum results, including in a data-dependent manner.

\section{Acknowledgments}

The authors are grateful to Dr. Keqi Tang and Dr. David A. Atkinson for insightful discussions of FAIMS and IMS. This research has been supported by Pacific Northwest National Laboratory (PNNL) Initiative for Explosives Detection and $\mathrm{NIH}$ National Center for Research Resources (RR 18522) located in the Environmental Molecular Sciences Laboratory, a national scientific user facility at PNNL sponsored by the U.S. Department of Energy (DOE) Office of Biological and Environmental Research. PNNL is operated for the DOE by Battelle under contract DEAC05-76RLO 1830.

\section{References}

1. Guevremont, R. High-Field Asymmetric Waveform Ion Mobility Spectrometry: A New Tool for Mass Spectrometry. J. Chromatogr. A 2004, 1058, 3-19.

2. Purves, R. W.; Guevremont, R. Electrospray Ionization High-Field Asymmetric Waveform Ion Mobility Spectrometry-Mass Spectrometry. Anal. Chem. 1999, 71, 2346-2357.

3. Guevremont, R.; Barnett, D. A.; Purves, R. W.; Vandermey, J. Analysis of a Tryptic Digest of Pig Hemoglobin Using ESI-FAIMS-MS. Anal. Chem. 2000, 72, 4577-4584.

4. Ells, B.; Barnett, D. A.; Purves, R. W.; Guevremont, R. Trace Level Determination of Perchlorate in Water Matrices and Human Urine Using ESI-FAIMS-MS. J. Environ. Monit. 2000, 2, 393-397.

5. Eiceman, G. A.; Tadjikov, B.; Krylov, E.; Nazarov, E. G.; Miller, R. A.; Westbrook, J.; Funk, P. Miniature Radio-Frequency Mobility Analyzer as a Gas Chromatographic Detector for Oxygen-Containing Volatile Organic Compounds, Pheromones, and Other Insect Attractants. J. Chromatogr. A 2001, 917, 205-217.

6. McCooeye, M. A.; Ding, L.; Gardner, G. J.; Fraser, C. A.; Lam, J.; Sturgeon, R. E.; Zoltan, M. Separation and Quantitation of the Stereoisomers of Ephedra Alkaloids in Natural Health Products Using Flow Injection-Electrospray Ionization-High-Field Asymmetric Waveform Ion Mobility Spectrometry-Mass Spectrometry. Anal. Chem. 2003, 75, 2538-2542.

7. Gabryelski, W.; Froese, K. L. Characterization of Naphthenic Acids by Electrospray Ionization High-Field Asymmetric Waveform Ion Mobility Spectrometry Mass Spectrometry. Anal. Chem. 2003, 75, 4612-4623.

8. Eiceman, G. A.; Tarassov, A.; Funk, P. A.; Hughs, S. E.; Nazarov, E. G.; Miller, R. A. Discrimination of Combustion Fuel Sources Using Gas Chromatography-Planar Field Asymmetric-Waveform Ion Mobility Spectrometry. J. Separation. Sci. 2003, 26, 585-593.

9. Buryakov, I. A. Express Analysis of Explosives, Chemical Warfare Agents and Drugs with Multicapillary Column Gas Chromatography and Ion Mobility Increment Spectrometry. J. Chromatogr. B 2004, 800, 75-82.

10. Li, J.; Purves, R. W.; Richards, J. C. Coupling Capillary Electrophoresis and High-Field Asymmetric Waveform Ion Mobility Spectrometry Mass Spectrometry for the Analysis of Complex Liposaccharides. Anal. Chem. 2004, 76, 4676-4683.

11. Eiceman, G. A.; Krylov, E.; Krylova, N.; Nazarov, E. G.; Miller, R. A. Separation of Ions from Explosives in Differential Mobility Spectrometry by Vapor-Modified Drift Gas. Anal. Chem. 2004, 76, 4937-4944.

12. Schmidt, H.; Tadjimukhamedov, F.; Mohrenz, I. V.; Smith, G. B.; Eiceman, G. A. Microfabricated Differential Mobility Spectrometry with Pyrolysis Gas Chromatography for Chemical Characterization of Bacteria. Anal. Chem. 2004, 76, 5208-5217.

13. Borysik, A. J. H.; Read, P.; Little, D. R.; Bateman, R. H.; Radford, S. E.; Ashcroft, A. E. Separation of $\beta_{2}$-Microglobulin Conformers by HighField Asymmetric Waveform Ion Mobility Spectrometry (FAIMS) Coupled to Electrospray Ionization Mass Spectrometry. Rapid Commun. Mass Spectrom. 2004, 18, 2229-2234.

14. McSheehy, S.; Mester, Z. Arsenic Speciation in Marine Certified Reference Materials Part I. Identification of Water-Soluble Arsenic Species Using Multidimensional Liquid Chromatography Combined with Inductively Coupled Plasma, Electrospray and Electrospray High-Field Asymmetric Waveform Ion Mobility Spectrometry with Mass Spectrometric Detection. J. Anal. Atom. Spectrom. 2004, 19, 373-380.

15. Barnett, D. A.; Ells, B.; Purves, R. W.; Guevremont, R. Application of ESI-FAIMS-MS to the analysis of tryptic peptides. J. Am. Soc. Mass Spectrom. 2002, 13, 1282-1291.

16. Venne, K.; Bonneil, E.; Eng, K.; Thibault, P. Improvement in Peptide Detection for Proteomics Analysis Using NanoLC-MS and High-Field Asymmetric Waveform Ion Mobility Spectrometry. Anal. Chem. 2005, 77, 2176-2186.

17. Tang, K.; Li, F.; Shvartsburg, A. A.; Strittmatter, E. F.; Smith, R. D. Two-Dimensional Gas-Phase Separations Coupled to Mass Spectrometry for Analyses of Complex Mixtures. Anal. Chem. 2005, 77, 6381-6388.

18. Shvartsburg, A. A.; Li, F.; Tang, K.; Smith, R. D. Characterizing the Structures and Folding of Free Proteins Using 2-D Gas-Phase Separations: Observation of Multiple Unfolded Conformers. Anal. Chem. 2006, 78, 3304-3315, 8575.

19. Shvartsburg, A. A.; Li, F.; Tang, K.; Smith, R. D. High-Resolution FAIMS Using New Planar Geometry Analyzers. Anal. Chem. 2006, 78, 37063714 .

20. Kapron, J.; Wu, J.; Mauriala, T.; Clark, P.; Purves, R. W.; Bateman, K. P. Simultaneous Analysis of Prostanoids Using Liquid Chromatography/High-Field Asymmetric Waveform Ion Mobility Spectrometry/Tandem Mass Spectrometry. Rapid Commun. Mass Spectrom. 2006, 20, 1504-1510.

21. Eiceman, G. A.; Karpas, Z. Ion Mobility Spectrometry; CRC: Boca Raton, FL, 2005.

22. Shvartsburg, A. A.; Tang, K.; Smith, R. D. Optimization of the Design and Operation of FAIMS Analyzers. J. Am. Soc. Mass Spectrom. 2005, 16, $2-12$.

23. Levin, D. S.; Miller, R. A.; Nazarov, E. G.; Vouros, P. Rapid Separation and Quantitative Analysis of Peptides Using a New NanoelectrosprayDifferential Mobility Spectrometer-Mass Spectrometer System. Anal. Chem. 2006, 78, 5443-5452.

24. Krylov, E. V. Comparison of the Planar and Coaxial Field Asymmetrical Waveform Ion Mobility Spectrometer (FAIMS). Int. J. Mass Spectrom. 2003, 225, 39-51.

25. Miller, R. A.; Zahn, M. Longitudinal Field Driven Field Asymmetric Ion Mobility Filter and Detection System. U.S. Patent 6512224, 2003.

26. Miller, R. A.; Zahn, M. Longitudinal Field Driven Ion Mobility and Detection System. U.S. Patent 6815669, 2004.

27. Javahery, G.; Thomson, B. A Segmented Radiofrequency-Only Quadrupole Collision Cell for Measurements of Ion Collision Cross Section on a Triple Quadrupole Mass Spectrometer. J. Am. Soc. Mass Spectrom. 1997, 8, 697-702.

28. Guo, Y.; Wang, J.; Javahery, G.; Thomson, B. A.; Siu, K. W. M. Ion Mobility Spectrometer with Radial Collisional Cooling. Anal. Chem. 2005, 77, 266-275.

29. Kim, T.; Tolmachev, A. V.; Harkewicz, R.; Prior, D. C.; Anderson, G.; Udseth, H. R.; Smith, R. D.; Bailey, T. H.; Rakov, S.; Futrell, J. H. Design and Implementation of a New Electrodynamic Ion Funnel. Anal. Chem. 2000, 72, 2247-2255.

30. Koeniger, S. L.; Merenbloom, S. I.; Valentine, S. J.; Jarrold, M. F.; Udseth, H. R.; Smith, R. D.; Clemmer, D. E. An IMS-IMS Analogue of MS-MS Anal. Chem. 2006, 78, 4161-4174.

31. Ibrahim, Y.; Tang, K.; Tolmachev, A. V.; Shvartsburg, A. A.; Smith, R. D. Improving Mass-Spectrometer Sensitivity Using a High-Pressure Electrodynamic Ion Funnel Interface. J. Am. Soc. Mass Spectrom. 2006, 17, 1299-1305.

32. Shvartsburg, A. A.; Tang, K.; Smith, R. D. Modeling the Resolution and Sensitivity of FAIMS Analyses. J. Am. Soc. Mass Spectrom. 2004, 15, 1487-1498.

33. Shvartsburg, A. A.; Tang, K.; Smith, R. D. FAIMS Operation for Realistic Gas Flow Profile and Asymmetric Waveforms Including Electronic Noise and Ripple. J. Am. Soc. Mass Spectrom. 2005, 16, 1447-1455.

34. Taraszka, J. A.; Kurulugama, R.; Sowell, R.; Valentine, S. J.; Koeniger, S. L.; Arnold, R. J.; Miller, D. F.; Kaufman, T. C.; Clemmer, D. E. Mapping the Proteome of Drosophila melanogaster: Analysis of Embryos and Adult Heads by LC-IMS-MS Methods. J. Proteome Res. 2005, 4, 1223-1237.

35. Ruotolo, B. T.; Gillig, K. J.; Woods, A. S.; Egan, T. F.; Ugarov, M. V.; Schultz, J. A.; Russell, D. H. Analysis of Phosphorylated Peptides by Ion Mobility-Mass Spectrometry. Anal. Chem. 2004, 76, 6727-6733.

36. Hilderbrand, A. E.; Myung, S.; Srebalus Barnes, C. A.; Clemmer, D. E. Development of LC-IMS-CID-TOFMS Techniques: Analysis of a 256 Component Tetrapeptide Combinatorial Library. J. Am. Soc. Mass Spectrom. 2003, 14, 1424-1436.

37. Jackson, S. N.; Wang, H.-Y. J.; Woods, A. S.; Ugarov, M.; Egan, T.; Schultz, J. A. Direct Tissue Analysis of Phospholipids in Rat Brain Using MALDI-TOFMS and MALDI-Ion Mobility-TOFMS. J. Am. Soc. Mass Spectrom. 2005, 16, 133-138.

38. Tempez, A.; Ugarov, M.; Egan, T.; Schultz, J. A.; Novikov, A.; Della-Negra, S.; Lebeyec, Y.; Pautrat, M.; Caroff, M.; Smentkowski, V. S.; Wang, H.-Y. J.; Jackson, S. N.; Woods, A. S. Matrix Implanted Laser Desorption Ionization (MILDI) Combined with Ion MobilityMass Spectrometry for Bio-Surface Analysis. J. Proteome Res. 2005, 4, $540-545$. 
39. Steiner, W. E.; Clowers, B. H.; Matz, L. M.; Siems, W. F.; Hill, H. H. Jr. Rapid Screening of Aqueous Chemical Warfare Agent Degradation Products: Ambient Pressure Ion Mobility Mass Spectrometry. Anal. Chem. 2002, 74, 4343-4352.

40. Kanu, A. B.; Haigh, P. E.; Hill, H. H. Surface Detection of Chemical Warfare Agent Simulants and Degradation Products. Anal. Chim. Acta 2005, 553, 148-159.

41. Guevremont, R.; Purves, R. W. Atmospheric Pressure Ion Focusing in a High-Field Asymmetric Waveform Ion Mobility Spectrometer. Rev. Sci. Instrum. 1999, 70, 1370-1383.

42. McDaniel, E. W.; Mason, E. A. Mobility and Diffusion of Ions in Gases; Wiley: NY, 1973.

43. Viehland, L. A.; Guevremont, R.; Purves, R. W.; Barnett, D. A. Comparison of High-Field Ion Mobility Obtained from Drift Tubes and a FAIMS Apparatus. Int. J. Mass Spectrom. 2000, 197, 123-130.

44. Shvartsburg, A. A.; Bryskiewicz, T.; Purves, R. W.; Tang, K.; Guevremont, R.; Smith, R. D. Field Asymmetric Waveform Ion Mobility Spectrometry Studies of Proteins: Dipole Alignment in Ion Mobility Spectrometry? J. Phys. Chem. B 2006, 110, 21966-21980.
45. Lin, S. N.; Griffin, G. W.; Horning, E. C.; Wentworth, W. E. Dependence of Polyatomic Ion Mobilities on Ionic Size. J. Chem. Phys. 1974, 60, 4994-4999.

46. Siems, W. F.; Wu, C.; Tarver, E. E.; Hill, H. H.; Larsen, P. R.; McMinn D. G. Measuring the Resolving Power of Ion Mobility Spectrometers. Anal. Chem. 1994, 66, 4195-4201.

47. Buryakov, I. A. Qualitative Analysis of Trace Constituents by Ion Mobility Increment Spectrometer. Talanta 2003, 61, 369-375.

48. Miller, R. A.; Eiceman, G. A.; Nazarov, E. G.; King, A. T. A Nove Micromachined High-Field Asymmetric Waveform-Ion Mobility Spectrometer. Sens. Actuat. B 2000, 67, 300-306.

49. Eiceman, G. A.; Krylov, E. V.; Tadjikov, B.; Ewing, R. G.; Nazarov, E. G. Miller, R. A. Differential Mobility Spectrometry of Chlorocarbons with a Micro-fabricated Drift Tube. Analyst, 2004, 129, 297-304.

50. Shvartsburg, A. A.; Mashkevich, S. V.; Smith, R. D. Feasibility of Higher-Order Differential Ion Mobility Separations Using New Asymmetric Waveforms. J. Phys. Chem. A 2006, 110, 2663-2673.

51. Verbeck, G. F.; Ruotolo, B. T.; Gillig, K. J.; Russell, D. H. Resolution Equations for High-Field Ion Mobility. J. Am. Soc. Mass Spectrom. 2004, $15,1320-1324$ 